\title{
PI Controller Design for Time Delay Systems Using an Extension of the Hermite-Biehler Theorem
}

\author{
Sami Elmadssia, Karim Saadaoui, and Mohamed Benrejeb \\ Unité de Recherche LARA-Automatique, Ecole Nationale d'Ingénieurs de Tunis, BP 37, le Belvédère, 1002 Tunis, Tunisia \\ Correspondence should be addressed to Sami Elmadssia; sami_elmadssia@yahoo.fr
}

Received 14 August 2012; Accepted 10 December 2012

Academic Editor: Alan Chan

Copyright (C) 2013 Sami Elmadssia et al. This is an open access article distributed under the Creative Commons Attribution License, which permits unrestricted use, distribution, and reproduction in any medium, provided the original work is properly cited.

We consider stabilizing first-order systems with time delay. The set of all stabilizing proportional-integral PI controllers are determined using an extension of the Hermite-Biehler theorem. The time delay is approximated by a second-order Padé approximation. For uncertain plants, with interval type uncertainty, robust stabilizing PI controllers are determined.

\section{Introduction}

In process control, many systems are represented by firstorder plants with time delay. Although several tuning rules are reported in the literature $[1,2]$, the problem of determining the entire set of stabilizing controllers of a given order, being PI or PID, for such systems is recently addressed in $[3,4]$. In [5], the Hermite-Biehler theorem is used to determine analytically the set of stabilizing gains $k_{p}, k_{i}$, and $k_{d}$ of a PID controller by replacing the time delay by a firstorder Padé approximation. In fact, extensions of the HermiteBiehler theorem were effectively used to determine the set of all stabilizing controllers of a given order and a given structure for systems without delay, see $[1,6,7]$.

In this paper, we use an extension of the Hermite-Biehler theorem to determine the set of all stabilizing PI controllers for a first-order system with time delay, where the time delay is replaced by a second-order Padé approximation. We show that for a fixed value of the proportional gain $k_{p}$, the set of stabilizing $k_{i}$ gains is a single interval. This conclusion still holds for higher-order Padé approximations. Next, we consider uncertain second-order systems with time delay and robust stabilizing PI controllers are determined.

The paper is organized as follows. In Section 2 we present some preliminary results which can be used to determine stabilizing proportional gains for systems without delay. These results are used in Section 3 to determine stabilizing PI controllers for first-order systems with time delay. In Section 4 robust stabilizing PI controllers are determined for uncertain systems. Illustrative examples are given in Section 5. Finally Section 6 contains some concluding remarks.

\section{Proportional Controllers}

In this section, an algorithm that determines the set of all proportional controllers [6] is reviewed. Let us first fix the notation used in this paper. Let $\mathbf{R}$ denote the set of real numbers and $\mathbf{C}$ denote the set of complex numbers and let $\mathbf{C}_{-}, \mathbf{C}_{0}, \mathbf{C}_{+}$denote the points in the open left-half, $j \omega$-axis, and the open right-half of the complex plane, respectively. Given a set of polynomials $\psi_{1}, \ldots, \psi_{1} \in \mathbf{R}[s]$ not all zero and $l>1$, their greatest common divisor is unique and it is denoted by $\operatorname{gcd}\left\{\psi_{1}, \ldots, \psi_{l}\right\}$. If $\operatorname{gcd}\left\{\psi_{1}, \ldots, \psi_{l}\right\}=1$, then we say $\left(\psi_{1}, \ldots, \psi_{l}\right)$ is coprime. The derivative of $\psi$ is denoted by $\psi^{\prime}$. The set $\mathscr{H}$ of Hurwitz stable polynomials are

$$
\mathscr{H}=\left\{\psi(s) \in \mathbf{R}[s]: \psi(s)=0 \Longrightarrow s \in \mathbf{C}_{-}\right\} .
$$

The signature $\sigma(\psi)$ of a polynomial $\psi \in \mathbf{R}[s]$ is the difference between the number of its $\mathbf{C}_{-}$roots and $\mathbf{C}_{+}$roots. Given $\psi \in$ $\mathbf{R}[s]$, the even-odd components $(a, b)$ of $\psi(s)$ are the unique polynomials $a, b \in \mathbf{R}[u]$ such that

$$
\psi(s)=a\left(s^{2}\right)+s b\left(s^{2}\right) .
$$

It is possible to state a necessary and sufficient condition for the Hurwitz stability of $\psi(s)$ in terms of its even-odd components $(a, b)$. Stability is characterized by the interlacing 
property of the real, negative, and distinct roots of the even and odd parts. This result is known as the HermiteBiehler theorem. Below is a generalization of the HermiteBiehler theorem applicable to not necessarily Hurwitz stable polynomials. Let us define the signum function $\mathcal{S}: \mathbf{R} \rightarrow$ $\{-1,0,1\}$ by

$$
\mathcal{S} r= \begin{cases}-1, & \text { if } r<0, \\ 0, & \text { if } r=0, \\ 1, & \text { if } r>0\end{cases}
$$

Lemma 1 (see [8]). Let a nonzero polynomial $\psi \in \mathbf{R}[s]$ have the even-odd components $(a, b)$. Suppose $b \neq 0$ and $(a, b)$ is coprime. Then, $\sigma(\psi)=r$ if and only if at the real negative roots of odd multiplicities $v_{1}>v_{2}>\cdots>v_{l}$ of $b$ the following holds:

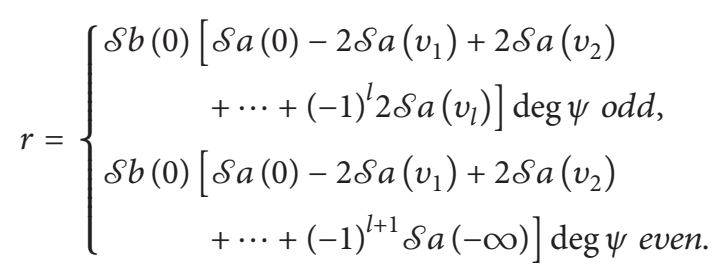

The following result determines the number of real negative roots of a real polynomial.

Lemma 2 (see [6]). A nonzero polynomial $\psi \in \mathbf{R}[s]$, such that $\psi(0) \neq 0$, has $r$ real negative roots without counting the multiplicities if and only if the signature of the polynomial $\psi\left(s^{2}\right)+s \psi^{\prime}\left(s^{2}\right)$ is $2 r$. All roots of $\psi$ are real, negative, and distinct if and only if $\psi\left(s^{2}\right)+s \psi^{\prime}\left(s^{2}\right) \in \mathscr{H}$.

We now describe a slight extension of the constant stabilizing gain algorithm of [8]. Given a plant

$$
g(s)=\frac{p(s)}{q(s)}
$$

where $p, q \in \mathbf{R}[s]$ are coprime with $m=\operatorname{deg} p$ less than or equal to $n=\operatorname{deg} q$, the set

$$
A_{r}(p, q):=\{k \in \mathbf{R}: \sigma[\phi(s, k)]=\sigma[q(s)+k p(s)]=r\}
$$

is the set of all real $k$ such that $\phi(s, k)$ has signature equal to $r$.

Let $(h, g)$ and $(f, e)$ be the even-odd components of $q(s)$ and $p(s)$, respectively, so that

$$
\begin{aligned}
& q(s)=h\left(s^{2}\right)+s g\left(s^{2}\right), \\
& p(s)=f\left(s^{2}\right)+s e\left(s^{2}\right) .
\end{aligned}
$$

Let $(H, G)$ be the even-odd components of $q(s) p(-s)$. Also let $F\left(s^{2}\right):=p(s) p(-s)$. By a simple computation, it follows that (we replace $s^{2}$ by $u$ ):

$$
\begin{gathered}
H(u)=h(u) f(u)-u g(u) e(u), \\
G(u)=g(u) f(u)-h(u) e(u), \\
F(u)=f^{2}(u)-u e^{2}(u) .
\end{gathered}
$$

With this setting, we have

$$
[q(s)+k p(s)] p(-s)=\left[H\left(s^{2}\right)+k F\left(s^{2}\right)\right]+s G\left(s^{2}\right) \text {. }
$$

If $G \equiv 0$ and if they exist, let the real negative roots with odd multiplicities of $G(u)$ be $\left\{v_{1}, \ldots, v_{l}\right\}$ with the ordering $v_{1}>$ $v_{2}>\cdots>v_{l}$, with $v_{0}:=0$ and $v_{l+1}:=-\infty$ for notational convenience.

The following algorithm determines whether $A_{r}(p, q)$ is empty or not and outputs its elements when it is not empty $[6]$.

Algorithm 3. (1) Consider all the sequences of signums

$$
\mathscr{I}= \begin{cases}\left\{i_{0}, i_{1}, \ldots, i_{l}\right\}, & \text { for odd } r-m, \\ \left\{i_{0}, i_{1}, \ldots, i_{l+1}\right\}, & \text { for even } r-m,\end{cases}
$$

where $i_{j} \in\{-1,1\}$ for $j=0,1, \ldots, l+1$.

(2) Choose all the sequences that satisfy

$$
r-\sigma(p)=\left\{\begin{array}{r}
i_{0}-2 i_{1}+2 i_{2}-2 i_{3}+\cdots+2(-1)^{l} i_{l} \\
\text { for odd } r-m, \\
i_{0}-2 i_{1}+2 i_{2}-2 i_{3}+\cdots+(-1)^{l+1} i_{l+1} \\
\text { for even } r-m .
\end{array}\right.
$$

(3) For each sequence of signums $\mathscr{I}=\left\{i_{j}\right\}$ that satisfy step (2), let

$$
\alpha_{\max }=\max \left\{-\frac{H}{F}\left(v_{j}\right)\right\}
$$

$$
\begin{array}{r}
\forall v_{j} \text { for which } F\left(v_{j}\right) \neq 0 \text { and } i_{j} \mathcal{S} F\left(v_{j}\right)=1, \\
\alpha_{\min }=\min \left\{-\frac{H}{F}\left(v_{j}\right)\right\}
\end{array}
$$

$\forall v_{j}$ for which $F\left(v_{j}\right) \neq 0$ and $i_{j} \mathcal{S F}\left(v_{j}\right)=-1$.

The set $A_{r}(p, q)$ is nonempty if and only if for at least one signum sequence $\mathscr{I}$ satisfying step (2), $\alpha_{\max }<\alpha_{\min }$ holds.

(4) $A_{r}(p, q)$ is equal to the union of intervals $\left(\alpha_{\max }, \alpha_{\min }\right)$ for each sequence of signums $\mathscr{I}$ that satisfy step (3).

The algorithm above is easily specialized to determine all stabilizing proportional controllers $c(s)=k$ for the plant $g(s)$. This is achieved by replacing $r$ in step (3) of the algorithm by $n$, the degree of $\phi(s, k)$.

Remark 4. By step (2) of Algorithm 3, a necessary condition for the existence of a $k \in A_{r}(p, q)$ is that the odd part of

$$
[q(s)+k p(s)] p(-s)
$$

has at least $\bar{r}=\max \{0,\lfloor(|r-\sigma(p)|-1) / 2\rfloor\}$ real negative roots with odd multiplicities. When solving a constant stabilization problem, this lower bound is $\bar{r}=\max \{0,\lfloor(n-\sigma(p)-1) / 2\rfloor\}$. 


\section{Stabilizing with PI Controllers}

In this section, we consider PI controllers

$$
c(s)=\frac{k_{p} s+k_{i}}{s}
$$

applied to a plant transfer function

$$
g(s)=\frac{1}{\tau s+a} e^{-\theta s}
$$

where $\tau>0$ is the time constant, $a$ is a constant whose sign and value determines the open-loop stability and steady-state gain, respectively, and $\theta$ represents the time delay. Our aim is to find all values of $\left(k_{p}, k_{i}\right)$ such that the closed-loop system is stable. We replace the time delay by a second-order Padé approximation

$$
e^{-\theta s} \approx \frac{\theta^{2} s^{2}-6 \theta s+12}{\theta^{2} s^{2}+6 \theta s+12}
$$

In what follows, we show how to find stabilizing values of $\left(k_{p}, k_{i}\right)$. The closed-loop characteristic polynomial is given by

$$
\phi\left(s, k_{p}, k_{i}\right)=q(s)+\left(k_{p} s+k_{i}\right) p(s),
$$

where

$$
\begin{gathered}
q(s)=s(\tau s+a)\left(\theta^{2} s^{2}+6 \theta s+12\right), \\
p(s)=\theta^{2} s^{2}-6 \theta s+12 .
\end{gathered}
$$

Multiplying $\phi\left(s, k_{p}, k_{i}\right)$ by $p(-s)$, we obtain

$$
\begin{aligned}
\psi\left(s, k_{p}, k_{i}\right)= & \phi\left(s, k_{p}, k_{i}\right) p(-s) \\
= & H\left(s^{2}\right)+k_{i} F\left(s^{2}\right) \\
& +s\left[G\left(s^{2}\right)+k_{p} F\left(s^{2}\right)\right] .
\end{aligned}
$$

Note here that $p(-s) \in \mathscr{H}$, therefore the odd part $G(u)+$ $k_{p} F(u)$ of $\psi(s)$ must have all its roots real and negative. The idea behind this method which determines the set of stabilizing parameters $\left(k_{p}, k_{i}\right)$ is to divide the problem into two subproblems: first we find all values of $k_{p}$ for which the polynomial $G(u)+k_{p} F(u)$ has all its roots real and negative. Next, by sweeping over all values of $k_{p}$ found in the first step and using Algorithm 3, we determine stabilizing values of $k_{i}$. In the first step, we construct a new polynomial

$$
\psi_{1}\left(s, k_{p}\right)=\left[G\left(s^{2}\right)+s G^{\prime}\left(s^{2}\right)\right]+k_{p}\left[F\left(s^{2}\right)+s F^{\prime}\left(s^{2}\right)\right] \text {, }
$$

using Lemma 2, finding values of $k_{p}$ such that $G(u)+k_{p} F(u)$ has all its roots real and negative is equivalent to stabilizing the new constructed polynomial $\psi_{1}\left(s, k_{p}\right)$. Hence our method consists of applying Algorithm 3 to two specially constructed plants:

$$
\begin{aligned}
g_{1}(s) & =\frac{p_{1}(s)}{q_{1}(s)} \\
& =\frac{F\left(s^{2}\right)+s F^{\prime}\left(s^{2}\right)}{G\left(s^{2}\right)+s G^{\prime}\left(s^{2}\right)}, \\
g_{2}(s) & =\frac{p_{2}(s)}{q_{2}(s)} \\
& =\frac{p(s)}{q(s)+k_{p} s p(s)} .
\end{aligned}
$$

Remark 5. For a fixed value of $k_{p}$, the set of stabilizing values of $k_{i}$, if they exist, are a single interval. In fact, using Padé approximation $p(-s)$ is always Hurwitz stable. By the interlacing property of the roots of the even and odd parts of a Hurwitz stable polynomial, it follows that only one sequence of signums satisfies step (2) in Algorithm 3 and therefore there is only one interval as a solution. This conclusion still holds if we use higher-order Padé approximations.

\section{Robust Stabilizing PI Controllers}

We now consider a second-order uncertain plant with an interval type uncertainty for the coefficients

$$
g(s)=\frac{\left[a_{2}^{-}, a_{2}^{+}\right] s^{2}+\left[a_{1}^{-}, a_{1}^{+}\right] s+\left[a_{0}^{-}, a_{0}^{+}\right]}{\left[b_{2}^{-}, b_{2}^{+}\right] s^{2}+\left[b_{1}^{-}, b_{1}^{+}\right] s+\left[b_{0}^{-}, b_{0}^{+}\right]} e^{-\theta s} .
$$

Our aim is to find robust stabilizing PI controllers. Replacing the time delay by a second-order Padé approximation, we get the following rational transfer function

$$
\begin{aligned}
g(s)= & \frac{p(s)}{q(s)} \\
= & \left(\left[c_{4}^{-}, c_{4}^{+}\right] s^{4}+\left[c_{3}^{-}, c_{3}^{+}\right] s^{3}+\left[c_{2}^{-}, c_{2}^{+}\right] s^{2}\right. \\
& \left.+\left[c_{1}^{-}, c_{1}^{+}\right] s+\left[c_{0}^{-}, c_{0}^{+}\right]\right) \\
& \times\left(\left[d_{4}^{-}, d_{4}^{+}\right] s^{4}+\left[d_{3}^{-}, d_{3}^{+}\right] s^{3}+\left[d_{2}^{-}, d_{2}^{+}\right] s^{2}\right. \\
& \left.\quad+\left[d_{1}^{-}, d_{1}^{+}\right] s+\left[d_{0}^{-}, d_{0}^{+}\right]\right)^{-1} .
\end{aligned}
$$

Let $p_{i}(s)$ and $q_{j}(s), i, j=1,2,3,4$ be the four Kharitonov polynomials corresponding to $p(s)$ and $q(s)$, respectively. Let $p_{i}^{\lambda}(s), i=1,2,3,4$ be the four Kharitonov segments of $p(s)$, that is,

$$
\begin{aligned}
& p_{1}^{\lambda}(s)=(1-\lambda) p_{1}(s)+\lambda p_{2}(s), \\
& p_{2}^{\lambda}(s)=(1-\lambda) p_{1}(s)+\lambda p_{3}(s), \\
& p_{3}^{\lambda}(s)=(1-\lambda) p_{2}(s)+\lambda p_{4}(s), \\
& p_{4}^{\lambda}(s)=(1-\lambda) p_{3}(s)+\lambda p_{4}(s),
\end{aligned}
$$


where $\lambda \in[0,1]$. The four Kharitonov segments $q_{j}^{\lambda}(s), j=$ $1,2,3,4$ of $q(s)$ can be defined similarly. Let $g_{\text {seg }}(s)$ denote the family of 32 segment plants

$$
\begin{gathered}
g_{\text {seg }}(s)=\left\{g_{i j}(s, \lambda) \mid g_{i j}(s, \lambda)=\frac{p_{i}^{\lambda}(s)}{q_{j}(s)}\right. \text { or } \\
g_{i j}(s, \lambda)=\frac{p_{i}(s)}{q_{j}^{\lambda}(s)}, i, j=1,2,3,4, \\
\lambda \in[0,1]\} .
\end{gathered}
$$

It is well known [9] that the family $g(s)$ is stabilized by a particular controller, if and only if the 32 segment plants $g_{\text {seg }}$ are stabilized by the same controller. Let $\widetilde{g}_{\text {seg }}(s)$ denote the family of 16 segment plants

$$
\begin{array}{r}
\tilde{g}_{\mathrm{seg}}(s)=\left\{g_{i j}(s, \lambda) \mid g_{i j}(s, \lambda)=\frac{p_{i}^{\lambda}(s)}{q^{j}(s)},\right. \\
i, j=1,2,3,4, \lambda \in[0,1]\} .
\end{array}
$$

It is shown in [10] that "the entire family $g(s)$ is stabilized by a particular PID controller, if and only if each segment plant $g_{i j}(s) \in \widetilde{g}_{\text {seg }}(s)$ is stabilized by that same PID controller." In reaching this result the structure of the PID controller was used to reduce the 32 segment plants to only 16. Since we are working with PI controllers, the numerator and denominator of the controller are convex directions [9]. Therefore, stabilizing the interval plant $g(s)$ by a PI controller is equivalent to stabilizing 16 vertex plants, namely,

$$
g_{v}(s)=\left\{g_{i j}(s) \mid g_{i j}(s)=\frac{p_{i}(s)}{q_{j}(s)}, i, j=1,2,3,4\right\} .
$$

The stabilizing controller, if any, can be determined by first calculating $k_{p}$ which is the intersection of $k_{p}$ 's found for the 16 plants mentioned above. We can then sweep over the values of $k_{p}$ and apply Algorithm 3 to the 16 vertex plants to find $k_{i}$.

\section{Application to the PT-326 Thermal Process}

An application example for the theoretical approaches presented in the precedent sections can be given by the temperature control of an air stream heater (process trainer PT-326), Figure 1. This type of process is found in many industrial systems such as furnaces, air conditioning, and so forth. The PT-326 Thermal Process Control models the industrial situation commonly found in such equipments as air conditioning plants where temperature control is achieved through a combination of more than one means. The process contained within the PT-326 comprises an air duct through which air may be circulated using an electrically driven variable speed fan. An electrically heated process block is

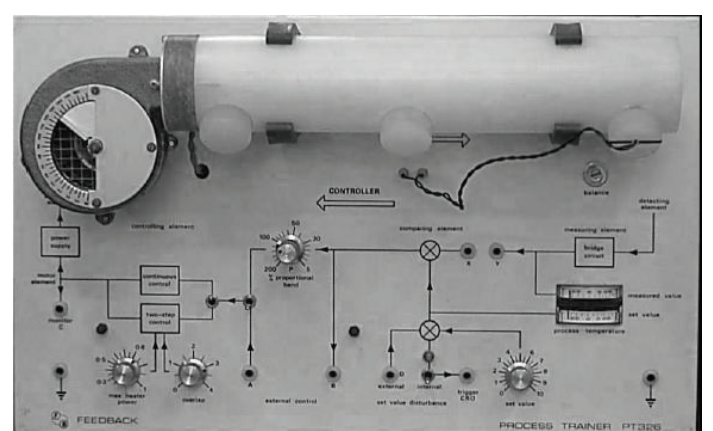

FIGURE 1: Front panel of the PT-326 apparatus.

mounted in the air flow path such that it attains thermal equilibrium by balancing the heat gained through the energy supplied to it via the heater coil and the heat lost through convection and conduction. Temperature control is achieved either by

(1) varying the heat energy input to the system by regulating electrical power to the heater coil or,

(2) varying the heat transfer rate by regulating the air flow rate either by

(i) controlling the speed of the circulating fan or,

(ii) restricting the actual flow channel itself using a controlled Vane mounted in the flow path.

Two platinum resistance thermometers $\left(T_{1}\right.$ and $\left.T_{2}\right)$ monitor the actual temperature of the block, being in the direct thermal contact with the block and being mounted on an insulation spacer to introduce thermal inertia and additional time constant effects into the control loop. Figure 1 below shows the front panel of the apparatus.

The physical principle which governs the behavior of the thermal process in the PT-326 apparatus is the balance of heat energy. The rate at which heat accumulates in a fixed volume $V$ enclosing the heater is

$$
q_{a}=q+q_{i}-q_{o}-q_{t},
$$

where $q$ is the rate at which heat is supplied by the heater, $q_{i}$ is the rate at which heat is carried into the volume $V$ by the coming air, $q_{o}$ is the rate at which heat is carried out of the volume $V$ by the outgoing air, and $q_{t}$ is the heat lost from the volume $V$ to the surroundings by radiation and conduction. Figure 2 below depicts the volume $V$. The behavior of the PT326 thermal process is governed by the balance of heat energy. When the air temperature inside the tube is supposed to be uniform, a linear delay system model can be obtained. Thus, the transfer function between the heater input voltage and the sensor output voltage can be obtained as $V_{o}(s) / V_{i}(s)=$ $k e^{-\theta s} /(\tau s+1)$ as shown in Figure 3.

A typical control objective in thermal systems is to maintain the temperature of some component at a user specified value called the set point. Figure 4 below depicts a closed-loop system designed to maintain the output temperature of the PT-326 apparatus at a desired set point. For the experiment, 


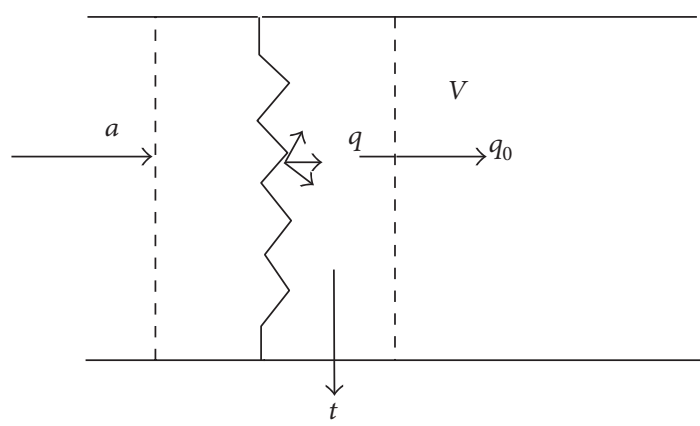

Figure 2: Heat transfer from the volume $V$.

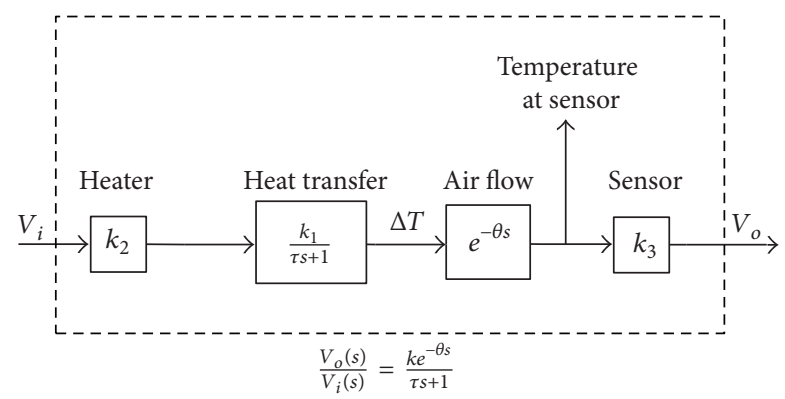

FIgURE 3: Block diagram of the PT-326 process.

the damper position is set to 30 , and the temperature sensor is placed in the third position. The transfer function is described by the following expression:

$$
g(s)=\frac{V_{o}(s)}{V_{i}(s)}=\frac{0.58 e^{-0.56 s}}{1.57 s+1} .
$$

5.1. Regions of All Stabilizing Values of $\left(k_{p}, k_{i}\right)$. Approximating the time delay by a second-order Padé approximation we get

$$
g(s)=\frac{0.58 s^{2}-6.214 s+22.19}{1.57 s^{3}+17.82 s^{2}+70.79 s+38.27} .
$$

The closed-loop characteristic polynomial is given by

$$
\begin{aligned}
\psi\left(s, k_{p}, k_{i}\right)= & s\left(1.57 s^{3}+17.82 s^{2}+70.79 s+38.27\right) \\
& +\left(k_{p} s+k_{i}\right)\left(0.58 s^{2}-6.214 s+22.19\right) .
\end{aligned}
$$

Using Lemma 2, we solve subproblem 1: find all values of $k_{p}$ such that $\psi\left(s, k_{p}\right)=20.0916 s^{4}+80.3664 s^{3}+857.5115 s^{2}+$ $1715 s+849.2113+k p\left(0.3364 s^{4}+1.3456 s^{3}-12.8734 s^{2}-\right.$ $25.7468 s+492.3961)$ is Hurwitz stable. Applying Algorithm 3, we get $k_{p} \in(-1.7247,9.855)$. The complete solution is given in Figure 5.

5.2. Simulation Results. In this section, we simulated the process PT-326 using MATLAB Simulink, we tested the evolution of output $y$ of the studied system in the presence of a disturbance. For the simulation, we took a step unit. We

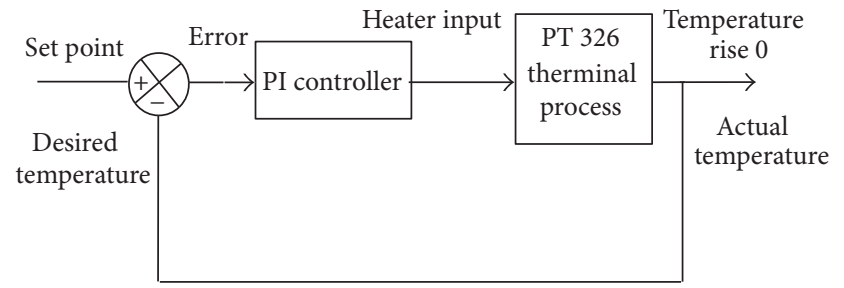

FIGURE 4: Closed-loop control of a thermal system.

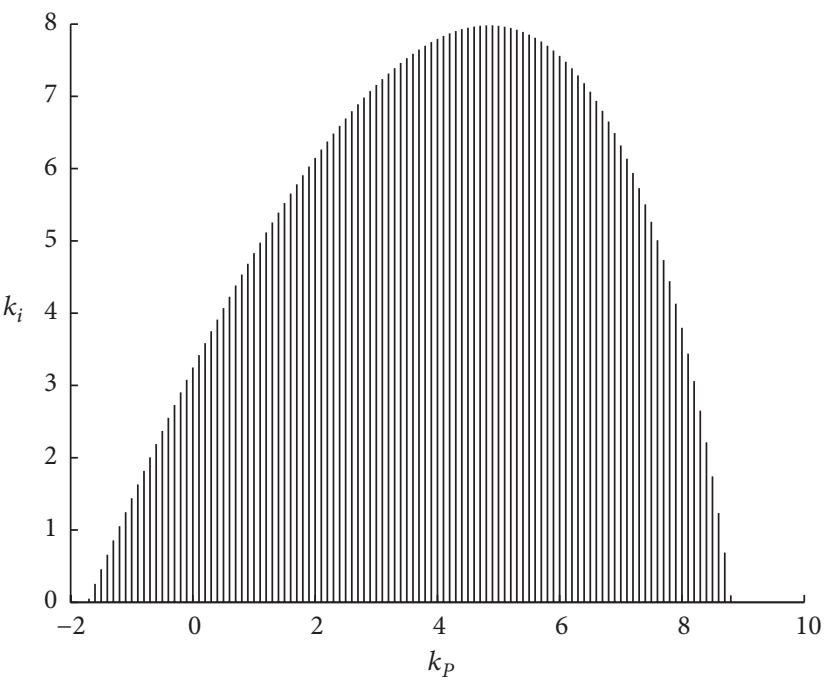

FIgURE 5: The stabilizing set of $\left(k_{p}, k_{i}\right)$ values.

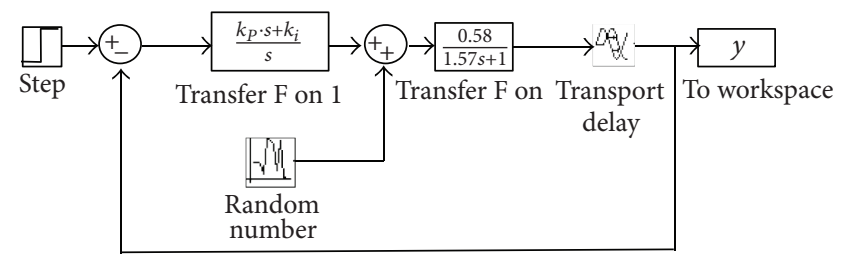

()) $\rightarrow t$

Clock To workspace

FIgURE 6: Simulink diagram of the system.

also took the block (Random Number) as a disturbance where its mean equal to 0 and variance equal to 0.01 and a simple time equal to 0.5. Figure 6 shows the MATLAB Simulink schematic of our application. Figure 7 shows the evolution of the output $y$ as a function of time. Notice that from this figure a choice of couple $\left(k_{p}, K_{i}\right)$ belonging the domain of stabilizing parameters can stabilize the studied system.

\section{Conclusions}

In this paper, we determine the set of all stabilizing PI controllers for first-order systems with time delay. By using a second-order Padé approximation for the time delay, we show that the set of stabilizing $k_{i}$ values is a single interval when $k_{p}$ is fixed. Robust stabilizing PI controllers 


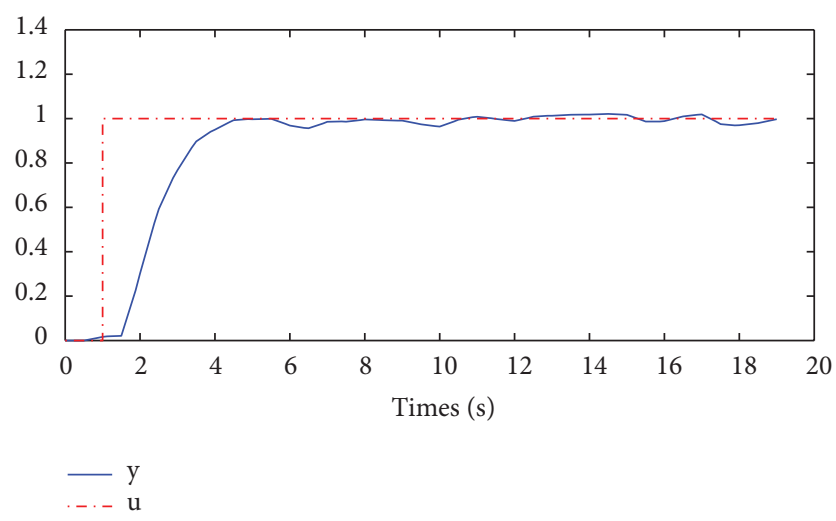

FIGURE 7: Simulation result for $k_{p}=1.8$ and $k_{i}=1.1$.

are determined for plants with uncertain parameters. Note that higher-order Padé approximations can be used without changing the analysis given in this paper, as the HermiteBiehler framework is applicable to plants irrelevant of the order.

\section{References}

[1] A. Datta, M. T. Ho, and S. P. Bhattacharyya, Structure and Synthesis of PID Controllers, Springer, New York, NY, USA, 2000.

[2] J. P. Richard, "Time-delay systems: an overview of some recent advances and open problems," Automatica, vol. 39, no. 10, pp. 1667-1694, 2003.

[3] G. J. Silva, A. Datta, and S. P. Bhattacharyya, "PI stabilization of first-order systems with time delay," Automatica, vol. 37, no. 12, pp. 2025-2031, 2001.

[4] G. J. Suva, A. Datta, and S. P. Bhattacharyya, "New results on the synthesis of PID controllers," IEEE Transactions on Automatic Control, vol. 47, no. 2, pp. 241-252, 2002.

[5] A. Roy and K. Iqbal, "PID controller design for first-order-plusdeadtime model via Hermite-Biehler theorem," in Proceedings of the American Control Conference, pp. 5286-5291, Denver, Colo, USA, June 2003.

[6] K. Saadaoui and A. B. Özgüler, "A new method for the computation of all stabilizing controllers of a given order," International Journal of Control, vol. 78, no. 1, pp. 14-28, 2005.

[7] K. Saadaoui and A. B. Özgüler, "On the set of all stabilizing first-order controllers," in Proceedings of the American Control Conference, Denver, Colo, USA, June 2003.

[8] A. B. Özgüler, A. A. Koçan et al., "An analytic determination of stabilizing feedback gains," Report 321, Institut für Dynamische Systeme Universität Bremen, 1994.

[9] B. R. Barmish, New Tools for Robustness of Linear Systems, Macmillan Publishing Company, 1994.

[10] N. Munro and M. T. Söylemez, "Fast calculation of stabilizing PID controllers of uncertain parameter systems," in Proceedings of ROCOND, Prague, Czech Republic, June 2000. 

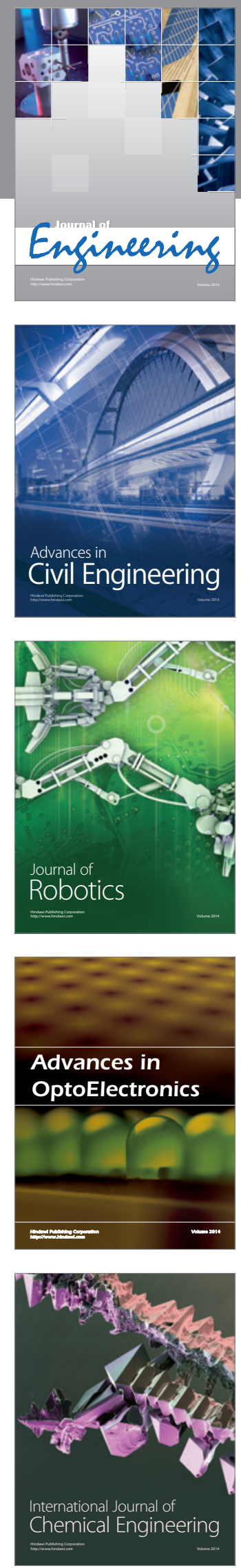

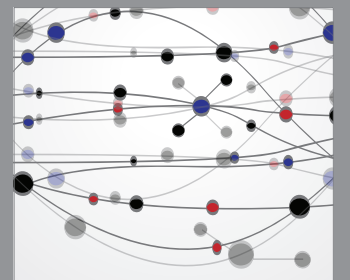

The Scientific World Journal
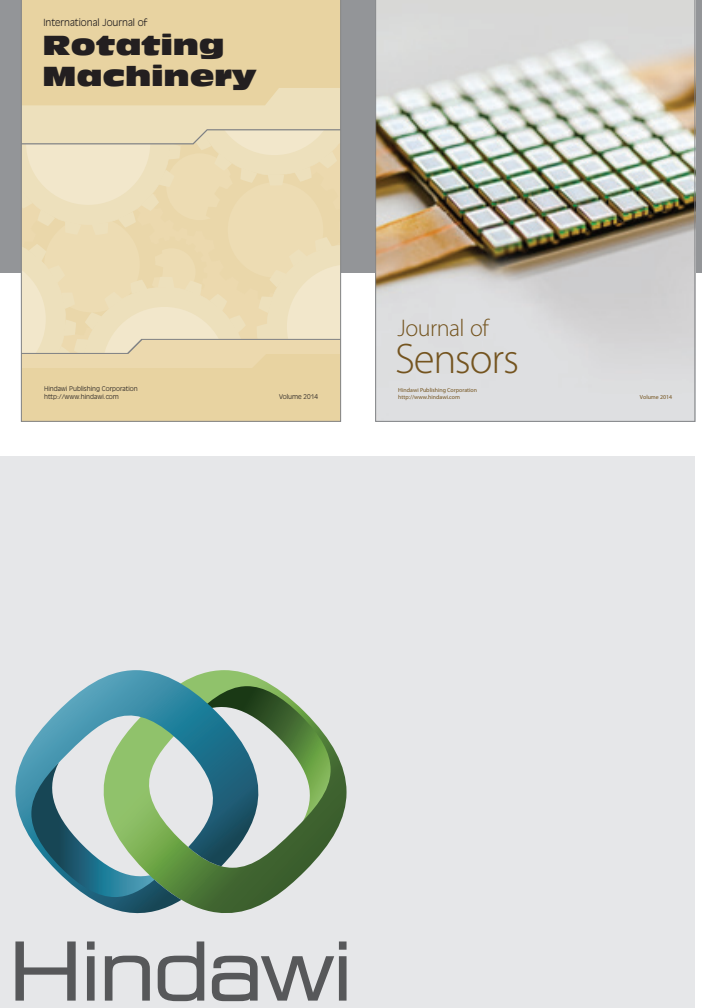

Submit your manuscripts at http://www.hindawi.com
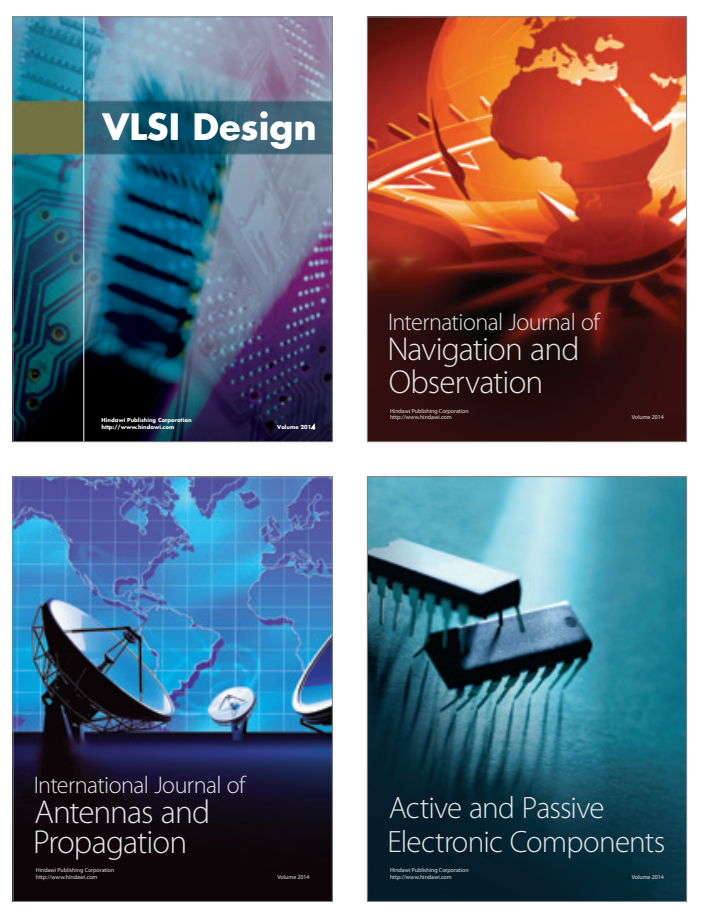
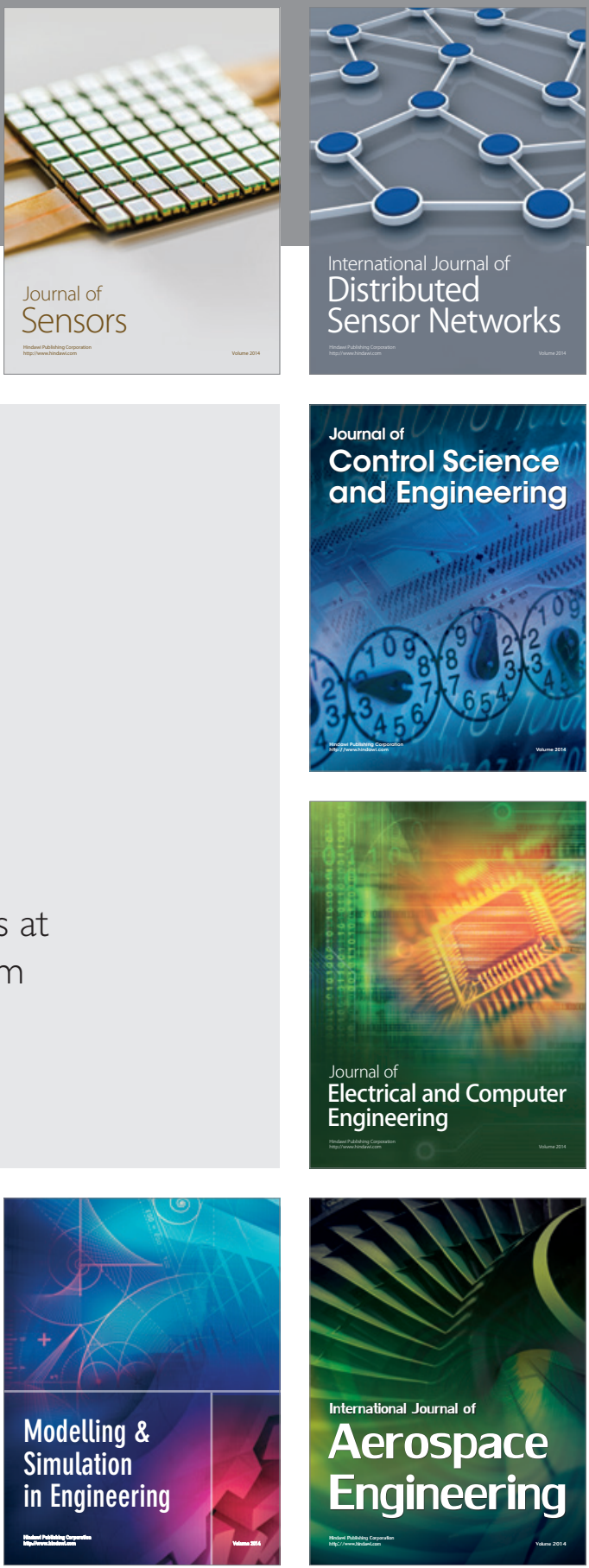

Journal of

Control Science

and Engineering
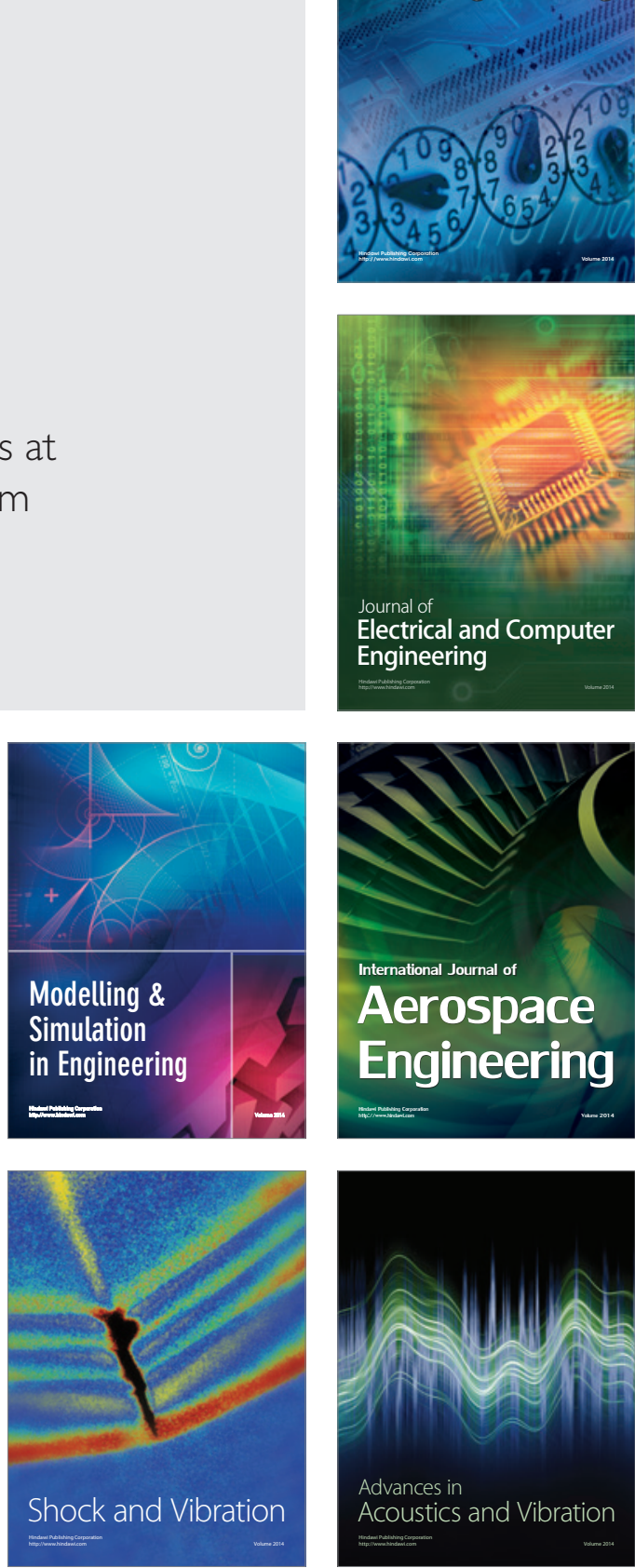